\title{
Dynamic Optimization of Chemical and Bio-chemical Processes using an Efficient Global Optimization Metaheuristic
}

\author{
J.A. Egea, E. Balsa-Canto, J.R. Banga, Instituto de Investigaciones Marinas (C.S.I.C.), Vigo, Spain
}

SNE Simulation Notes Europe SNE 19(3-4), 2009, 41-46, doi: 10.11128/sne.19.tn.09949

A metaheuristic based on scatter search for global dynamic optimization of chemical and bio-chemical processes is presented. It is designed to overcome typical difficulties of nonlinear dynamic systems optimization such as noise, flat areas, non-smoothness and/or discontinuities. It balances between intensification and diversification by coupling a local search procedure with a global search and makes use of memory to avoid simulations in previously explored areas. Its application to three dynamic optimization case studies proves its efficiency and robustness, showing also a very good scalability.

\section{Introduction}

Dynamic optimization (or open loop optimal control) appears in many industrial applications to optimize a pre-defined performance index (e.g., profitability) subject to some specifications over a time interval. Objective functions and/or constraints formulated from mathematical models describing industrial processes are usually highly nonlinear, which often causes non-convexity. Besides, non-smoothness and discontinuities can be present, thus the use of global optimization methods is needed for many dynamic optimization problems from this field [7]. In recent years, a special class of stochastic global optimization methods called metaheuristics, which provide excellent solutions (often the global optimum) in relatively short computation times, has appeared.

Scatter search is a population-based metaheuristic introduced by Glover [12] which combines a global phase with an intensification method, usually a local search [16]. Compared to other evolutionary or genetic algorithms, scatter search has a small population (called reference set or RefSet) consisting of high quality and diverse solutions which are systematically combined. Our scatter search-based algorithm has been written in Matlab under the name SSm. This study goes beyond a simple exercise of applying scatter search to dynamic optimization problems, but presents innovative mechanisms to obtain a good balance between intensification and diversification in a short-term search horizon. In many instances, dynamic optimization problems are non-convex and multimodal, thus the use of global optimization techniques becomes crucial for solving them [7]. The application of our algorithm for solving nonlinear optimization problems arising from chemical and biological systems has provided excellent results $[10,18]$.
This paper is organized as follows: Section 1 states the problem of dynamic optimization. Our algorithm is depicted in Section 2. Section 3 presents the three case studies used in this paper for our experiments as well as the results obtained. The paper finishes with some conclusions.

\section{Dynamic optimization: problem statement}

The general dynamic optimization problem has the following mathematical form:

$$
\min _{\boldsymbol{u}(t), \boldsymbol{v}, t_{f}} C\left(\boldsymbol{x}\left(t_{f}\right), \boldsymbol{z}\left(t_{f}\right), \boldsymbol{u}\left(t_{f}\right), \boldsymbol{v}, t_{f}\right)
$$

subject to the system's dynamics:

$$
\begin{aligned}
& \boldsymbol{F}\left(\dot{\boldsymbol{x}}, \boldsymbol{x}_{t}, \boldsymbol{x}_{\xi}, \boldsymbol{x}_{\xi \xi}, \boldsymbol{x}(t), \boldsymbol{z}\left(t_{f}\right), \boldsymbol{u}\left(t_{f}\right), \boldsymbol{v}, t_{f}\right)=\mathbf{0} \\
& \boldsymbol{x}(0)=\boldsymbol{x}_{0} \\
& \boldsymbol{u}(0)=\boldsymbol{u}_{0} \\
& \boldsymbol{z}(0)=\boldsymbol{z}_{0} \\
& \boldsymbol{x}(\boldsymbol{\Omega})=\boldsymbol{x}_{\boldsymbol{\Omega}}
\end{aligned}
$$

where $x_{t}=\partial x / \partial t, \quad x_{\xi}=\partial x / \partial \xi, \quad x_{\xi \xi}=\partial^{2} x / \partial \xi^{2}$; $\boldsymbol{x}(t) \in \boldsymbol{X} \subset \boldsymbol{R}^{n l+n d}$ (with $n l=$ number of lumped state variables and $n d=$ number of distributed state variables) and $\boldsymbol{z}(t) \in \boldsymbol{Z} \subset \boldsymbol{R}^{m}$ are the vectors of differential and algebraic states respectively; $\boldsymbol{u}(t) \in$ $\boldsymbol{U} \subset \boldsymbol{R}^{p}$ is the vector of control (input) variables; $\boldsymbol{v}(t) \in \boldsymbol{V} \subset \boldsymbol{R}^{q}$ are time invariant parameters; $t$ is the time (and tf is the final time); $C$ is a functional to be minimized; $\boldsymbol{F}$ is the set of partial differentialalgebraic equations describing the systems dynamics; finally, $\boldsymbol{x}_{0}, \boldsymbol{z}_{0}$, and $\boldsymbol{u}_{0}$ are the values of the respective vectors at the initial time $t_{0}$, and $\boldsymbol{x}_{\boldsymbol{\Omega}}$ is the value of $\boldsymbol{x}$ at spatial boundary.

Equality and inequality constraints may be imposed. Some of them must be satisfied over the whole process time (path constraints), 


$$
\begin{aligned}
& \boldsymbol{H}_{\mathrm{path}}\left(\boldsymbol{x}\left(t_{f}\right), \boldsymbol{z}\left(t_{f}\right), \boldsymbol{u}\left(t_{f}\right), \boldsymbol{v}, t_{f}\right)=0 \quad \forall t \\
& \boldsymbol{G}_{\mathrm{path}}\left(\boldsymbol{x}\left(t_{f}\right), \mathbf{z}\left(t_{f}\right), \boldsymbol{u}\left(t_{f}\right), \boldsymbol{v}, t_{f}\right)=0 \quad \forall t
\end{aligned}
$$

while others must be only satisfied at the end of the process (endpoint constraints),

$$
\begin{aligned}
& \boldsymbol{H}_{\text {end }}\left(\boldsymbol{x}\left(t_{f}\right), \boldsymbol{z}\left(t_{f}\right), \boldsymbol{u}\left(t_{f}\right), \boldsymbol{v}, t_{f}\right)=0 \quad \forall t \\
& \boldsymbol{G}_{\text {end }}\left(\boldsymbol{x}\left(t_{f}\right), \boldsymbol{z}\left(t_{f}\right), \boldsymbol{u}\left(t_{f}\right), \boldsymbol{v}, t_{f}\right)=0 \quad \forall t
\end{aligned}
$$

The control variables and/or the time-invariant parameters may be subject to lower and upper bounds:

$$
\begin{aligned}
& \boldsymbol{u}^{\mathrm{lb}} \leq \boldsymbol{u}(t) \leq \boldsymbol{u}^{\mathrm{ub}} \\
& \boldsymbol{v}^{\mathrm{lb}} \leq \boldsymbol{v}(t) \leq \boldsymbol{v}^{\mathrm{ub}}
\end{aligned}
$$

In practice, the dynamic optimization of distributed systems typically involves transforming the original system into an equivalent lumped system and applying lumped-system dynamic optimization methods. Therefore, a spatial discretization approach is usually used to transform the original infinite dimension partial differential equations (PDE) into a large-scale, and possibly stiff, set of ordinary differential equations (ODEs) [4]. The accurate solution of the resulting ODE system then often requires the use of an implicit ODE solver.

In this work we will consider the CVP approach [22] using the Piecewise Constant approximation, PC (i.e., zero order polynomial) with fixed-length time intervals. Different number of intervals will be used for each problem in order to check the scalability of the different optimization methods.

\section{A scatter search algorithm for dynamic optimization of chemical and bioprocesses}

\subsection{Diversification Generation Method}

$S S m$ begins by generating an initial set of diverse vectors in the search space. The method makes use of memory taking into account the number of times that every decision variable appears in different parts of the search space [13].

\subsection{Initial RefSet formation}

For building the initial RefSet, after generating the set of diverse solutions, a subset of high quality and diverse points is selected. The first step consists in evaluating all diverse vectors and selecting some of them in terms of quality. The RefSet is completed with the remaining diverse vectors by maximizing the minimum Euclidean distance to the included vectors in the RefSet.

\subsection{Subset Generation and Solution Combination methods}

After the initial RefSet is built, its solutions are sorted according to their quality and we apply the Subset Generation Method. In our implementation, it consists in selecting all pairs of solutions in the RefSet to combine them. To avoid repeating combinations with the same pair of solutions, we use a memory term which keeps track of the pairs previously combined. Regarding the Solution Combination Method, we use a type of combination based on hyper-rectangles [21], which enhances the diversification. Depending on their position in the RefSet every pair of combined solutions may generate from two up to four new solutions.

\section{$2.4 \quad$ Updating the RefSet}

As recommended by Laguna and Marti [17], we update the RefSet considering the quality of the elements. This strategy may cause convergence to sub-optimal solutions or stagnation of the search in flat areas. To avoid these effects, we have implemented two filters [10] which restrict the incorporation of solutions that contribute only slight diversity to the RefSet.

\subsection{Improvement method}

The Improvement Method consists in a local search, selecting the initial points by means of different filters. In this work, we have considered a gradientbased method [11] and the Nelder and Mead method implemented in Matlab [1]. In applications related to chemical and bioprocess engineering, we often face time-consuming evaluation problems or complex topologies which can make the local search inefficient. This implies that the application of the Improvement Method should be restricted to a low number of promising solutions. Here we use merit and distance heuristic filters introduced by Ugray et al. [21] to avoid performing local searches from poor quality solutions or from solutions which are likely to provide already found local minima.

\subsection{RefSet rebuilding}

Due to the memory term which avoids combinations between RefSet members previously combined, the optimization procedure may stop if no new solutions enter the RefSet in a given iteration. Advanced scatter search designs overcome this problem by resorting a mechanism to partially rebuild the RefSet. The method is usually the same as that used to create the initial RefSet, in the sense that it uses the max-min distance criterion for selecting diverse solutions. 


\begin{tabular}{c|c|c|c|c|c|c|c}
$\boldsymbol{\rho}$ & & CMAES & DE & glcDirect & OQNLP & SRES & SSm \\
\hline \multirow{4}{*}{$\mathbf{1 0}$} & Best & $\mathbf{2 0 3 1 6 . 1 1}$ & $\mathbf{2 0 3 1 6 . 0 8}$ & 20203.74 & $\mathbf{2 0 3 1 6 . 1 1}$ & 20305.96 & $\mathbf{2 0 3 1 6 . 1 1}$ \\
\cline { 2 - 8 } & Mean & 19889.67 & 20100.72 & - & - & 20093.14 & 20291.38 \\
\cline { 2 - 8 } & Worst & 18996.02 & 19672.46 & - & - & 19554.01 & 20192.48 \\
\hline \multirow{2}{*}{$\mathbf{2 0}$} & Best & $\mathbf{2 0 4 1 2 . 1 4}$ & 20404.36 & 19738.01 & $\mathbf{2 0 4 1 2 . 1 9}$ & 20327.11 & $\mathbf{2 0 4 1 2 . 1 9}$ \\
\cline { 2 - 8 } & Mean & 20273.76 & 20383.95 & - & - & 20237.58 & 20412.19 \\
\cline { 2 - 8 } & Worst & 19953.39 & 20341.29 & - & - & 20095.71 & 20412.19 \\
\hline \multirow{2}{*}{$\mathbf{4 0}$} & Best & 20430.84 & 20375.32 & 19544.88 & $\mathbf{2 0 4 4 4 . 4 7}$ & 20214.40 & $\mathbf{2 0 4 4 4 . 8 6}$ \\
\cline { 2 - 8 } & Mean & 20360.73 & 20239.27 & - & - & 19726.07 & 20444.86 \\
\cline { 2 - 7 } & Worst & 20110.08 & 19902.08 & - & - & 19466.64 & 20444.86
\end{tabular}

Table 1. Results for the ethanol production problem.

\begin{tabular}{c|c|c|c|c|c|c|c}
$\boldsymbol{\rho}$ & & CMAES & DE & glcDirect & OQNLP & SRES & SSm \\
\hline \multirow{4}{*}{$\mathbf{1 0}$} & Best & $\mathbf{8 7 . 9 3 4}$ & $\mathbf{8 7 . 9 3 4}$ & 87.258 & 87.775 & $\mathbf{8 7 . 9 2 7}$ & $\mathbf{8 7 . 9 3 1}$ \\
\cline { 2 - 8 } & Mean & 87.837 & 87.914 & - & - & 87.688 & 87.906 \\
\cline { 2 - 8 } & Worst & 87.340 & 87.835 & - & - & 87.348 & 87.889 \\
\hline \multirow{2}{*}{$\mathbf{2 0}$} & Best & 87.948 & $\mathbf{8 8 . 0 1 3}$ & 84.490 & 87.400 & 87.671 & 87.998 \\
\cline { 2 - 8 } & Mean & 87.841 & 87.955 & - & - & 86.900 & 87.885 \\
\cline { 2 - 8 } & Worst & 87.599 & 87.767 & - & - & 85.064 & 87.796 \\
\hline \multirow{4}{*}{$\mathbf{4 0}$} & Best & 87.914 & 87.926 & 80.657 & 87.547 & 82.709 & $\mathbf{8 7 . 9 9 9}$ \\
\cline { 2 - 8 } & Mean & 87.861 & 87.802 & - & - & 82.709 & 87.863 \\
\cline { 2 - 8 } & Worst & 87.745 & 87.565 & - & - & 82.709 & 87.595
\end{tabular}

Table 2. Results for the penicillin production problem.

We propose an alternative strategy to maximize the number of search directions.

In this strategy, the vectors refilling the RefSet are chosen to maximize the number of relative directions defined by them and the existing vectors in the RefSet [10].

\subsection{Intensification strategies}

One of the filters mentioned above may prevent the search from focusing on intensification, especially during the first iterations.

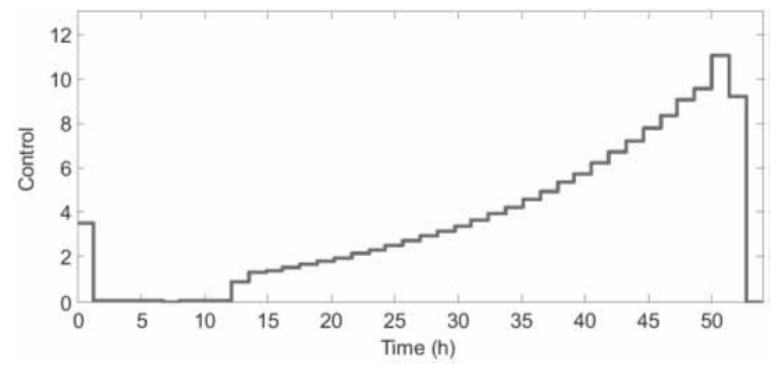

Figure 1. Optimal control profile for the ethanol production problem $(\rho=40)$.
To allow combinations between high quality solutions (which do not apply to enter the RefSet because of the distance filter) and RefSet members, we store the solutions which can not enter the RefSet but have a better function value than the second RefSet member.

These stored solutions are then combined with the best RefSet solution, increasing the probability of obtaining high quality solutions by combination in early stages of the search [10]. Another advanced strategy (the go beyond strategy) to enhance the intensification of the search has been implemented in our algorithm. It consists in exploiting promising directions [9].

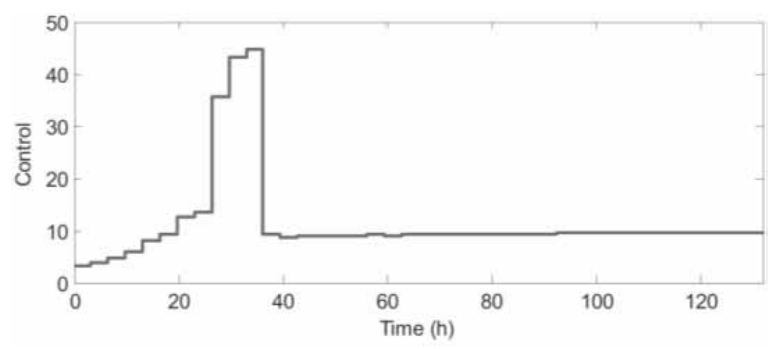

Figure 2. Optimal control profile for the penicillin production problem $(\rho=40)$ 


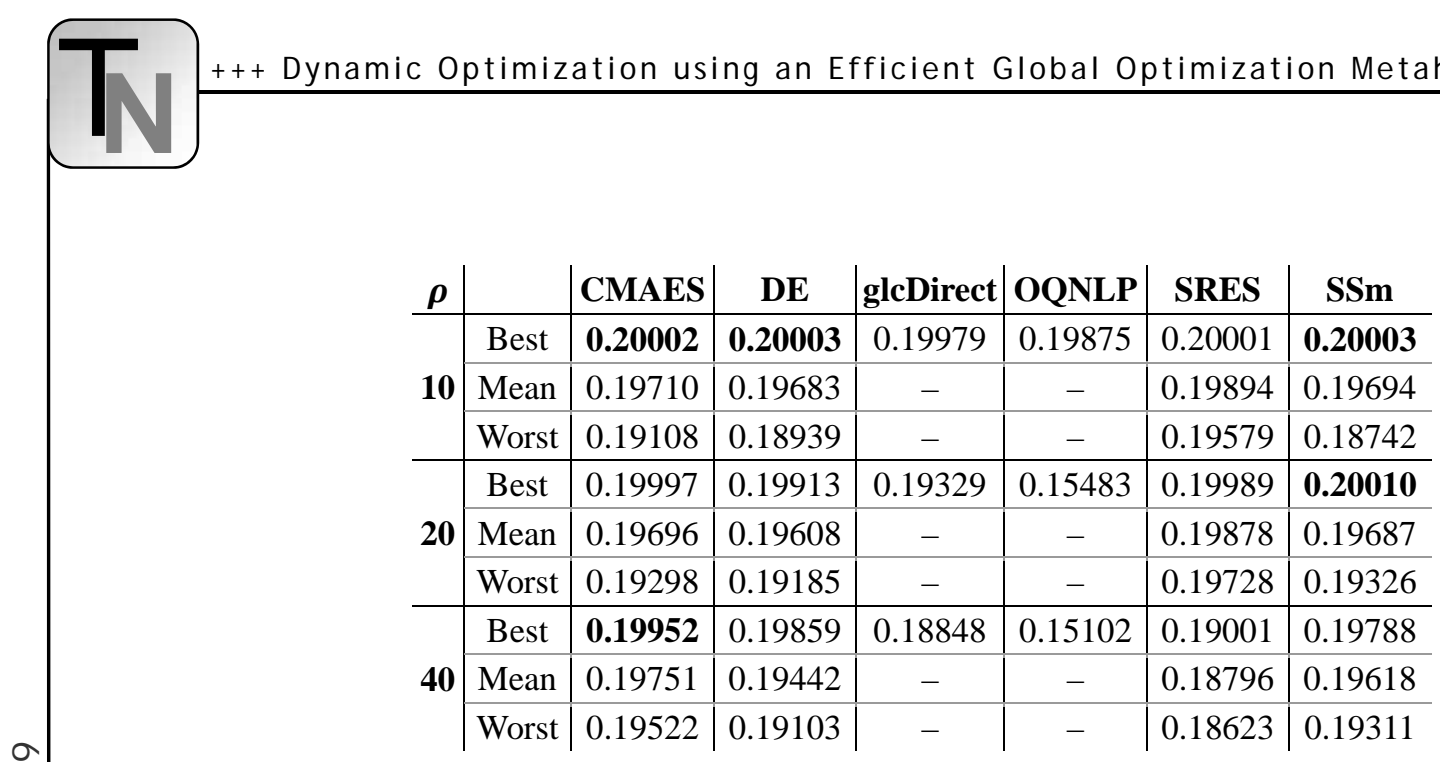

Table 3. Results for the drying process problem.

\section{Computational experiments}

In this section, a set of bioprocess dynamic optimization problems will be used as case studies to test the performance of the algorithm proposed in this work. A set of different state-of-the-art global optimization methods has been selected to compare their results with those obtained with the algorithm proposed in this study: CMAES [2], DE [20], SRES [19], DIRECT [14] and $O Q N L P$ [15]. Regarding stochastic solvers, ten runs were performed for each problem.

\subsection{Ethanol production in a fed-batch reactor}

This system is a fed-batch bioreactor for the production of ethanol [6]. The objective is to find the feed rate which maximizes the yield of ethanol. Table 1 presents results for every solver with the different levels of discretization considered. Figure 1 presents the optimal control profile for the highest level of discretization.

\subsection{Penicillin production in a fed-batch fermenter}

This problem deals with the dynamic optimization of a fed-batch fermenter for the production of penicillin [6]. The optimal control problem is to maximize the total amount of penicillin produced using the feed rate of substrate as the control variable.

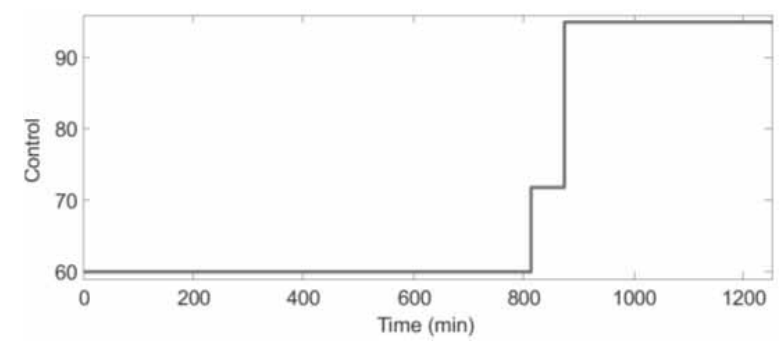

Figure 3. Optimal control profile for the drying process problem $(\rho=40)$
In our experiments, $S S m$ provided the best solution for the levels of discretization $\rho=10,40$. Table 2 presents results for every solver with the different levels of discretization and Figure 2 presents the optimal control profile for the highest level of discretization.

\subsection{Drying operation}

In this section we consider a food convective drying problem, similar to the one formulated by Banga and Singh [5]. The aim is to dry a cellulose slab maximizing the retention of a nutrient.

The dynamic optimization problem associated with the process consists of finding the dry bulb temperature along the time to maximize the nutrient retention at the final time. Table 3 presents results for every solver with the different levels of discretization and Figure 3 presents the optimal control profile for the highest level of discretization.

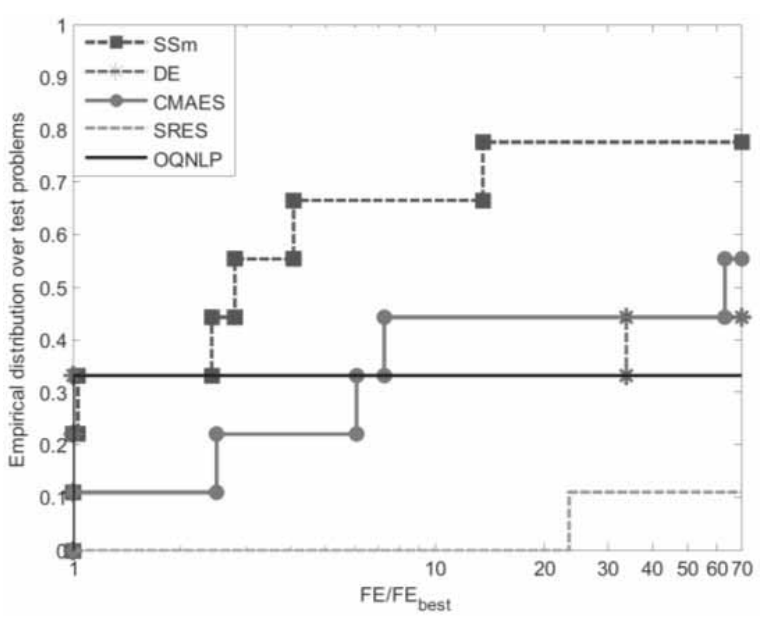

Figure 4. Performance profiles 


\section{Executive summary of results}

In this section we provide a summary of the results obtained in this work by making use of the performance profiles methodology [8]. Following [3], we define the success performance $\boldsymbol{F} \boldsymbol{E}$ for a solver on a specific problem by:

$$
\boldsymbol{F} \boldsymbol{E}=\text { eval }_{\text {mean }} \frac{\# \text { all runs (10) }}{\# \text { successful runs }}
$$

where a run is considered successful if it obtained the optimal solution with a relative error $\leq 0.01 \%$ (in our problems, we consider it as the best solution found by any of the solvers). With this definition, the best success performance $\boldsymbol{F} \boldsymbol{E}_{\text {best }}$ is given by the lowest value of $\boldsymbol{F} \boldsymbol{E}$ for every problem. Figure 4 shows the empirical distribution function of the success performance $\boldsymbol{F} \boldsymbol{E} / \boldsymbol{F} \boldsymbol{E}_{\text {best }}$ over all the problems. As shown in the performance profiles, $S S m$ solves the highest percentage of problems compared with rest of solvers tested.

\section{Conclusions}

We have developed a scatter search-based methodology which intends to be effective for solving global dynamic optimization problems from the biotechnological and food industries. The procedure treats the objective function as a black box, making the search algorithm context-independent. We have expanded and advanced knowledge associated with the implementation of scatter search procedures.

We have tested the proposed methodology over a set of dynamic optimization problems from the biotechnological and food industries. In order to have an idea about their efficiency, they have been compared with other state-of-the-art global optimization methods. The results obtained showed that the proposed methodology is adequate for the kind of problems intended to solve. In all cases our algorithm was competitive, providing the best solution among the tested solvers in many of the examples.

It is to note that the algorithm's behaviour is not affected by the problem size since it provides excellent results for every level of discretization considered in this study.

\section{Acknowledgements}

The authors acknowledge financial support from EU project BaSysBio LSHG-CT-2006-037469 and Spanish MICINN project MultiSysBio DPI2008-06880-C03-02.

\section{References}

[1] The MathWorks Inc: Optimization Toolbox for Use with Matlab. User's guide. Version 2.

[2] Auger, A. and Hansen, N.: A restart CMA evolution strategy with increasing population size. In: Proc. IEEE Congress on Evolutionary Computation, IEEE CEC 2005, 1769-1776.

[3] Auger, A. and Hansen, N.: Performance evaluation of an advanced local search evolutionary algorithm. In: Proc. IEEE Congress on Evolutionary Computation, IEEE CEC 2005, 1777-1784.

[4] Balsa-Canto, E., Banga, J.R., Alonso, A.A. and Vassiliadis, V.S.: Dynamic optimization of distributed parameter systems using second-order directional derivatives, Industrial \& Engineering Chemistry Research, 43 (2004), 6756-6765.

[5] Banga, J.R. and Paul Singh, R.: Optimization of air drying of foods, Journal of Food Engineering, 23 (1994), 189-211.

[6] Banga, J.R., Alonso, A.A. and Singh, R.P.: Stochastic dynamic optimization of batch and semicontinuous bioprocesses, Biotechnology Progress, 13 (1997), 326-335.

[7] Banga, J.R., Balsa-Canto, E., Moles, C.G. and Alonso, A.A.: Dynamic optimization of bioprocesses: Efficient and robust numerical strategies, Journal of Biotechnology, 117 (2005), 407-419.

[8] Dolan, E.D. and More, J.J.: Benchmarking optimization software with performance profiles, Mathematical Programming, Series B, 91 (2002), 201-213.

[9] Egea, J.A.: New Heuristics for Global Optimization of Complex Bio-Processes, PhD Thesis, University of Vigo, 2008.

[10] Egea, J.A., Rodriguez-Fernandez, M., Banga, J. R., Marti, R.: Scatter search for chemical and bioprocess optimization, Journal of Global Optimization, 37 (2007), 481-503.

[11] Exler, O. and Schittkowski, K.: A trust region $S Q P$ algorithm for mixed-integer nonlinear programming, Optimization Letters, 1 (2007), 269-280.

[12] Glover, F.: Heuristics for Integer Programming Using Surrogate Constraints, Decision Sciences, 8 (1977), 156-166.

[13] Glover, F., Laguna, M., Marti, R.: Scatter search. In: Advances in Evolutionary Computation: Theory and Applications, (Eds.A. Ghosh, Tsutsui, S.), SpringerVerlag, New York, 2003, 519-537.

[14] Jones, D.R.: DIRECT global optimization algorithm. In: Encyclopedia of Optimization, (Eds.C. A. Floudas and P. M. Pardalos), Kluwer Academic Publishers, Dordrecht, 2001, 431-440.

[15] Laguna, M. and Marti, R.: The OptQuest Callable Library. In: Optimization software class libraries, (Eds. S. Voss and D. L. Woodruff), Kluwer Academic Publishers, Boston, 2002, 193-218. 
[16] Laguna, M. and Marti, R.: Scatter search : methodology and implementations in $C$. Kluwer Academic Publishers, Boston, 2003

[17] Laguna, M. and Marti, R.: Experimental testing of advanced scatter search designs for global optimization of multimodal functions, Journal of Global Optimization, 33 (2005), 235-255.

[18] Rodriguez-Fernandez, M., Egea, J. A., Banga, J. R.: Novel Metaheuristic for Parameter Estimation in Nonlinear Dynamic Biological Systems, BMC Bioinformatics, 7 (2006), 483.

[19] Runarsson, T.P. and Yao, X.: Stochastic ranking for constrained evolutionary optimization, IEEE Transactions on Evolutionary Computation, 4 (2000), 284-294.

[20] Storn, R. and Price, K.: Differential evolution - A simple and efficient heuristic for global optimization over continuous spaces, Journal of Global Optimization, 11 (1997), 341-359.
[21] Ugray, Z., Lasdon, L., Plummer, J., Glover, F., J., K. and Marti, R.: A Multistart Scatter Search Heuristic for Smooth NLP and MINLP Problems. In: Metaheuristic optimization via memory and evolution: tabu search and scatter search, (Eds.C. Rego and B. Alidaee), Kluwer Academic Publishers, Norwell, Mass., 2005, 25-58.

[22] Vassiliadis, V.S., Sargent, R.W.H. and Pantelides, C.C.: Solution of a class of multistage dynamic optimization problems. Problems without path constraints, Industrial and Engineering Chemistry Research, 33 (1994), 2111-2122. 1593

\section{Corresponding author: J.R. Banga}

Process Engineering Group

Instituto de Investigaciones Marinas (C.S.I.C.)

Eduardo Cabello, 6, 36208, Vigo, Spain

julio@iim.csic.es

Received \& Accepted: MATHMOD 2009

Revised: September 25, 2009

Accepted: October 20, 2009 